\title{
Confirmation of deafness in infancy
}

\author{
P M Watkin, M Baldwin
}

\begin{abstract}
Aim-To assess delay in confirming hearing impairment in infants identified by universal neonatal screening and to investigate the causes.

Patients-Infants identified from 25199 babies screened from January 1992 to December 1997.

Methods-A two stage transient evoked oto-acoustic emission test (TEOAE), with a threshold auditory brainstem response (ABR) recording undertaken on those who failed. The screen identified infants with a permanent congenital hearing impairment (PCHI) averaging $40 \mathrm{dBnHL}$ or worse in the best ear. Those with less impairment were also ascertained. The positive predictive value (PPV) of the ABR test and measures of delay between identification and eventual diagnosis were analysed.
\end{abstract}

Results-A targeted PCHI was found in $1.18 / 1000$ neonates. The PPV of the ABR for confirming a targeted PCHI was $100 \%$ when the ABR threshold was $\geqslant 80 \mathrm{dBnHL}$. Nine of 11 infants with this threshold had severe or profound permanent deafness. The delay from ABR to audiological certainty was about 1 month-diagnosis was confirmed around 3 months. There was uncertainty when the ABR was 40-80 dBnHL. The PPV was $60 \%$ and $8 \%$ when the $A B R$ thresholds were $70 \mathrm{dBnHL}$ and $50 \mathrm{dBnHL}$, respectively. 85 of 111 infants with ABR thresholds in this range had a temporary conductive impairment. Their early diagnosis depended upon the type and degree of hearing impairment and diagnosis was delayed to about 8 months in these infants.

Conclusions-Hearing impairments identified by universal screening are delayed in all but those with severe or profound bilateral PCHI. This delay can be reduced by applying in early infancy a battery of audiological tests and requires further exploration.

(Arch Dis Child 1999;81:380-389)

Keywords: transient evoked oto-acoustic emission test; deafness; screening; confirmation of deafness

The identification of children with a permanent congenital hearing impairment (PCHI) by universal neonatal screening has been recommended by an NHS health technology assessment critical review. ${ }^{1}$ Two districts in the UK and many in the USA have implemented such screens. Their experiences have confirmed that they can be practicable and effective, ${ }^{2-4}$ and the national screening committee is considering their wider introduction in the UK. However, their worth depends upon much more than the implementation of a successful screen. This is just the first link in a chain of multidisciplinary endeavour aiming to improve outcomes for those with congenital deafness. Effective and timely habilitation cannot be assumed to follow automatically from early detection.

Some of the difficulties encountered in providing early habilitation have been investigated by the Whipps Cross Hospital universal screen. It aims to identify those infants with a permanent congenital hearing impairment of moderate or worse degree $(>40 \mathrm{dBHL}$ in the better hearing ear). The screen was one of the first programmes to use transient evoked otoacoustic emissions (TEOAEs) and has been implemented as a routine service in the East London district of Waltham Forest since 1992. ${ }^{34}$ To date, over 35000 neonates have been screened.

However, despite the success of the screen, it would be naive to think that the outcome has improved for all those infants identified. Delays have still occurred in the introduction of habilitation for some of the infants. Investigation of an initial three year cohort confirmed several inter-related causes for these delays. ${ }^{5}$ Deafness can present with great subtlety and, not unreasonably, parents may reject hearing aid fitting unless they recognise deafness to be present. In addition, the misgivings of parents might be compounded by the "audiological uncertainties" of the professionals. Those guiding and recommending interventions might themselves be unsure that an infant failing the screen has a targeted PCHI requiring long term habilitation.

Such diagnostic uncertainties are not surprising. Persistent absence of TEOAEs confirms the presence of a cochlear or middle ear dysfunction (or both), but does not differentiate between the two. Yet, when targeting only those with a permanent impairment, it is necessary to exclude those whose hearing loss is caused by middle ear effusion. Unfortunately, there is no simple way of doing this at this age. Otoscopic examination is extremely 


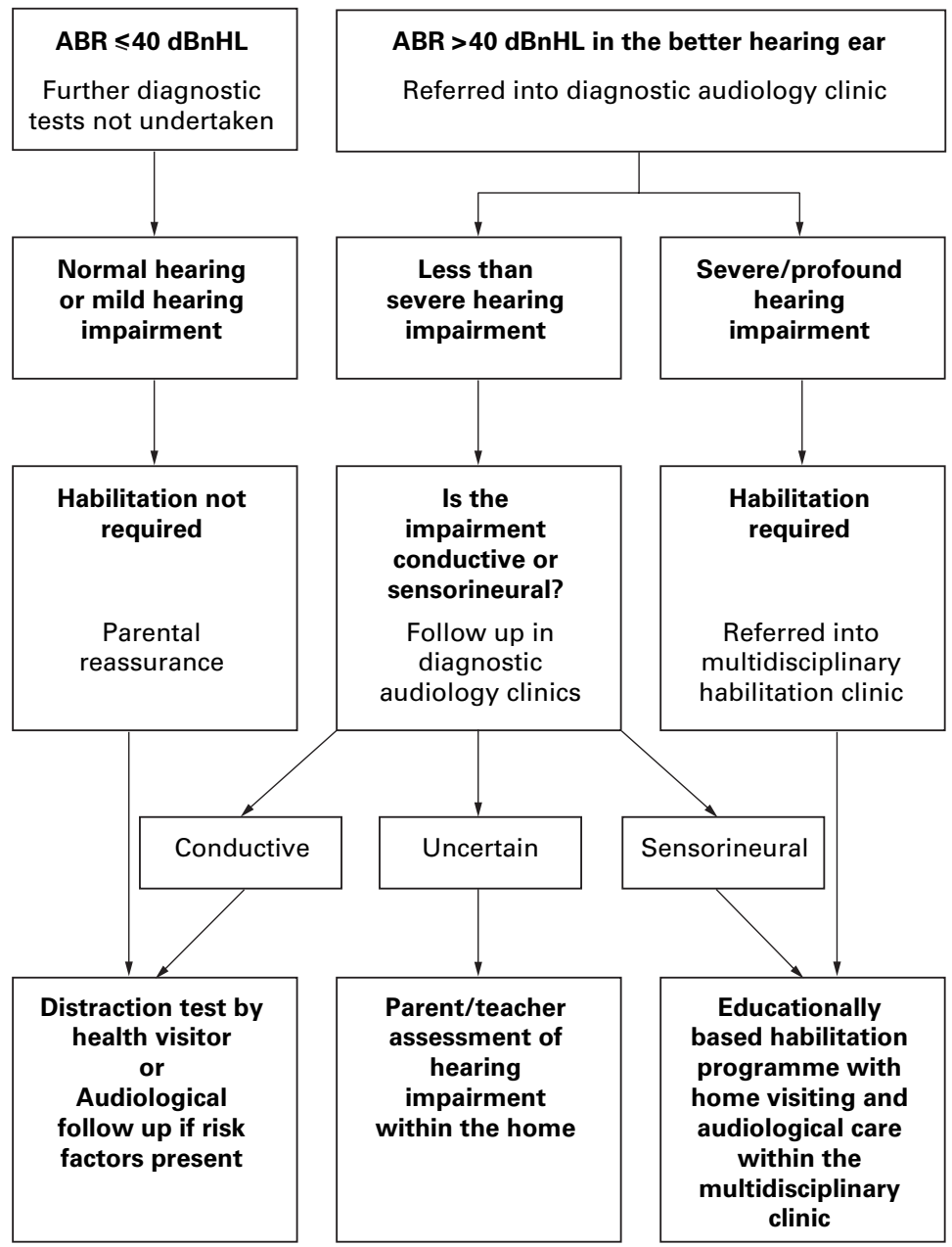

Figure 1 Management strategies after the auditory brainstem response (ABR) test.

difficult and often inaccurate ${ }^{67}$ and, although conventional tympanometry with a $220 \mathrm{~Hz}$ probe tone is still used to determine the presence of middle ear abnormalities in the neonate, ${ }^{8}$ the use of higher frequency (678 and $1000 \mathrm{~Hz}$ ) probe tones has also been recommended. ${ }^{910}$ Unfortunately, these probe tone frequencies are not available on most tympanometers. Therefore, clinical uncertainties in confirming middle ear effusions persist.

This is not the only problem confronting audiological diagnosis at this age. The assessment of the degree of hearing impairment in itself is difficult. This is not measured by the TEOAE screening test but requires an auditory brainstem response (ABR) measurement. This is an electrophysiological test of brainstem function and does not assess hearing perception. For over two decades it has been the "gold standard" for measuring hearing thresholds in early infancy. However, the conventional test uses "click" stimuli to elicit the brainstem response and, although on average these correlate with the hearing between 2000 and $4000 \mathrm{~Hz}$, this might not be so for an individual infant. ${ }^{11}$ However, frequency specific stimuli to obtain frequency specific ABR thresholds have generally not been used in the UK. Therefore, the usual ABR offers a narrow diagnostic view, and the results need supplementing by obser- vations of behavioural responses to a variety of sounds. Such observations are invariably made in the home by the parents, and are formalised in the clinical setting as behavioural observation audiometry. Unfortunately, reflexive behaviours to loud sounds are usually simple to elicit, but observation of attentive behaviours to quiet sounds are required to assess thresholds, and these responses are subtle and deceptively difficult to assess at this age. ${ }^{12}$ Therefore, the confirmation of the hearing level also presents as yet unresolved difficulties.

How important are these diagnostic uncertainties for universal neonatal screening? Middle ear disease is common, but often asymptomatic in early infancy. ${ }^{13}$ Will hard pressed audiology services be swamped by babies who have failed the screen, but in whom audiological diagnosis is uncertain? Do these difficulties delay habilitation so that the usefulness of the screen is compromised? To examine these questions, we investigated the diagnostic processes undertaken on those infants identified by the Whipps Cross universal neonatal screen from January 1992 to December 1997.

\section{Subjects and methods}

THE SCREENING PROGRAMME

During the period under investigation, the Whipps Cross programme combined two stage TEOAE testing followed by air conduction ABR for TEOAE failures. An initial TEOAE test was undertaken whenever possible before discharge from the maternity unit. Those missed, and those born out of the district or at home returned for their initial test in the hospital audiology department. All bilateral failures of the initial TEOAE tests were given an appointment for a TEOAE retest in the audiology department. Bilateral failure at the retest prompted an obligatory $\mathrm{ABR}$. In line with the aims of the screen, those failing in one ear were only retested if the parents wanted the further test. The ABR was also offered when parental anxiety indicated an early diagnostic test, or when a paediatrician was concerned that the neonate might have an auditory neuropathy. The ABR was undertaken to threshold in $10 \mathrm{dBnHL}$ steps.

\section{MANAGEMENT AFTER THE ABR}

Figure 1 summarises the management strategies after the ABR. In line with the programme aims, those babies who had failed the TEOAE screen in both ears, but who had an ABR threshold $\leqslant 40 \mathrm{dBnHL}$ were considered to have "passed" the screen and their parents were strongly reassured. They were appointed for behavioural localisation tests at 8 months. These infant distraction tests ${ }^{14}$ were undertaken by both the audiologists and the health visitors.

Those infants with a $>40 \mathrm{dBnHL}$ threshold in the better hearing ear were referred to diagnostic assessment clinics undertaken by the district's senior audiologists. Medical and birth histories were obtained and the infant was physically and audiologically examined. Further ABR tests were undertaken when required to confirm the initial result, or if it was thought 
that the $\mathrm{ABR}$ threshold might have changed with increased maturation. A picture of the overall hearing ability was achieved by behavioural observation audiometry. ${ }^{12}$ Both reflexive and attentive responses were observed, with speech and narrow bands of noise as principal stimuli. Babies were examined by auroscopy and tests of middle ear function (tympanometry) were undertaken. For the period detailed in this study tympanometry was undertaken using a Grason-Stadler GSI 28A automated tympanometer. This equipment uses a $226 \mathrm{~Hz}$ probe tone and sweeps between a pressure range of +200 and $-400 \mathrm{daPa}$. During the past year or so of the study period a GSI 33 tympanometer was also used. Although this uses multifrequency probe tones, and a wider pressure range, interpretation of the results was the subject of further study, and we report the results achieved with the GSI 28A. Parents were also encouraged to observe hearing responses within the home and to evaluate these responses with reference to a checklist provided. ${ }^{15}$

The immediate management of those assessed in the diagnostic clinics differed according to the degree of hearing impairment found on assessment. With confirmation of a severe or profound PCHI, the infant was assigned to a weekly multidisciplinary habilitation clinic undertaken jointly with the teacher of the deaf. Refinements of the audiological diagnosis were undertaken as part of the ongoing habilitation and hearing aid fitting programme. The teacher visited these infants and hearing aids were fitted as early as possible. Management of those who had a $>40 \mathrm{dBnHL}$ ABR threshold in the better hearing ear, but where the diagnostic assessments had demonstrated a less than severe degree of deafness, was more cautious. Initially, greater emphasis was placed on the diagnostic assessment, with the audiological and otological examination aimed at assessing whether the hearing loss was permanent or attributable to middle ear effusion. As soon as there was "audiological certainty" that these infants had a PCHI, they were referred to the multidisciplinary habilitation clinics. When there was doubt about the permanence of the deafness (and therefore the need for habilitation), the teacher of the deaf visited their home to observe the baby's responses with parents and to evaluate the functional importance of the hearing impairment. Subsequently, follow up was in the diagnostic audiology clinics and habilitation started as soon as there was agreement that it was required.

\section{THE COHORT STUDIED}

From the introduction of the universal neonatal screen in January 1992 until the end of December 1997, 25199 neonates were screened. At the time of the study, the youngest cohort member had passed through infancy, and the oldest had been in primary school for over one year. Over this period, 28890 were eligible for programme entry, and coverage rose during the first nine months. Thereafter, $93.6 \%$ of those eligible received an initial TEOAE test (23 673 of 25 287). This six year cohort of 25199 neonates who had received the TEOAE tests was the subject of our study.

THE DIAGNOSTIC ASSESSMENTS

The number of $A B R$ tests

We identified how many infants were referred from the TEOAE tests for an ABR along with the reasons for referral. Although the protocol indicated an ABR when the TEOAE had been failed on two occasions, we encouraged reactivity. An immediate ABR was accepted for late entrants to the screen, and appointment defaulters, or where there was professional or parental concern. These ABR results were ascertained for the threshold achieved in the better hearing ear when it had been undertaken for bilateral TEOAE failure, and for the worse hearing ear when it had been prompted by unilateral failure. The infants were categorised into groups according to the $\mathrm{ABR}$ thresholds. Three ABR categories were used: $\leqslant 40 \mathrm{dBnHL} ;>40 \mathrm{dBnHL}$ and $<80 \mathrm{dBnHL}$; $\geqslant 80 \mathrm{dBnHL}$

The ABR referrals and the results were also examined longitudinally from 1993 to 1997 (1992 was omitted because acceptable coverage was only achieved in the later months). This longitudinal analysis was required to measure the effect of programme changes undertaken during the study period. After a detailed analysis of the first three year results, the stringent TEOAE pass/fail criteria were relaxed. The less stringent screen criteria were used for the final two years of the study period. The longitudinal analysis was undertaken by ascertaining the annual yield of ABRs with the thresholds aggregated into the three groups given above. Differences in the annual results were analysed by means of the $\chi^{2}$ test.

The positive predictive value of the ABR tests Those children with a PCHI were ascertained and classified according to the British Society of Audiology descriptors of degree of hearing impairment (table 1). Although those children with a mild or unilateral deafness were not targeted for detection, inevitably some were picked up by the screen. We investigated the value of the initial $A B R$ threshold in predicting the presence of a PCHI. This positive predictive value (PPV) was evaluated for those failing the TEOAE test bilaterally and unilaterally. Because the youngest in the cohort have only just reached 1 year of age, it is possible that a small number of those who failed the TEOAE tests and had an ABR have not had their targeted PCHI diagnosed with certainty yet. Although all those with an ABR threshold in the better hearing ear $\leqslant 40 \mathrm{dBnHL}$ were appointed for an infant distraction test, coverage of this test at 8 months was incomplete. It is very likely that most of those with a mild PCHI will not be identified until school age, whether or not they failed the TEOAE component of the neonatal screen. Therefore, we quantified the PPV of the ABR for confirming the presence of a targeted PCHI, but not the PPV of the ABR for confirming a mild PCHI. The PPV for unilateral PCHIs was also a problem. After ABR, reliable audiometric 
Table 1 The audiometric descriptors of hearing impairment (British Society of Audiology, 1988)

\begin{tabular}{ll}
\hline Descriptor & Threshold \\
\hline Mild impairment & $21-40 \mathrm{dBnHL}$ \\
Moderate impairment & $41-70 \mathrm{dBnHL}$ \\
Severe impairment & $71-95 \mathrm{dBnHL}$ \\
Profound impairment & $>95 \mathrm{dBnHL}$ \\
\hline
\end{tabular}

separation is only possible with the completion of a masked audiogram. Behavioural tests without the use of ear inserts or headphones do not allow unilateral confirmation with certainty, although it can often be suspected from the test battery. Unilateral ABR failures who were also thought clinically to have a unilateral PCHI were ascertained in calculating the PPV.

Delayed "audiological certainty"

Ultimately, the value of early identification must be assessed from improvements in outcome in terms of reduction in disability, but for evaluating hearing screens intermediate outcome indicators are used as surrogates. ${ }^{15}$ In our study, the surrogate outcome indicators used were the age of the initial TEOAE failure (age of identification), the age of ABR confirmation, and the age of audiological certainty. We defined "certainty" as the age at which both the type and degree of hearing impairment were confirmed. Cumulative distributions of these outcome ages were constructed for the infants identified with a targeted PCHI. We omitted those obligatory referrals with external canal atresia, or those identified by professional or parental concerns, those who had been transferred to other central

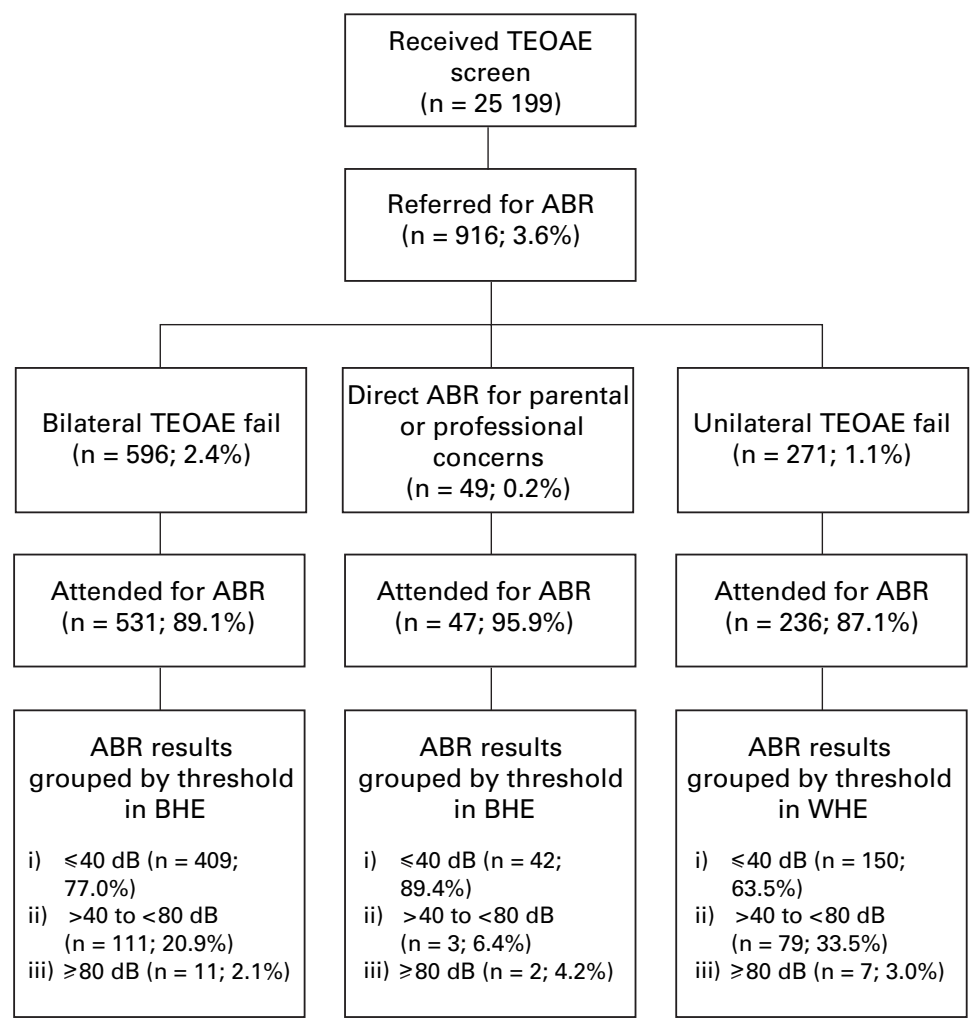

Figure 2 The auditory brainstem response (ABR) tests and the results from 1992 to 1997. BHE, better hearing ear; TEOAE, transient evoked oto-acoustic emission test; WHE, worst hearing ear.
London audiology departments, and those who died in infancy. Therefore, the cumulative distributions reflected the results of the Whipps Cross screen with the intermediate outcome ages up to the end of infancy available.

The delays from age of identification by the TEOAE screen to confirmation of ABR threshold, and from the ABR to the age at which audiological certainty was achieved, were measured for each child with $>40$ $\mathrm{dBnHL}$ bilateral ABR impairment. The infants were also subdivided according to the presence or not of a PCHI and the degree of hearing impairment confirmed with certainty by the diagnostic assessments. The degrees were classified according to the British Society of Audiology descriptors of degree of hearing impairment (table 1). Delays to the age of audiological certainty were measured and comparisons between the groups were made by transforming the delays to ranks, and applying a Mann Whitney U test. We also investigated the delay in achieving certainty for those with a targeted PCHI who had passed the ABR. For the reasons detailed above, we considered only those unilateral PCHIs with a reliably masked pure tone audiogram were to have been diagnosed with audiological certainty when assessing the unilateral outcome delays.

\section{The results of tympanometry}

We ascertained those who were referred to the diagnostic clinics with a $>40$ dBnHL ABR threshold, but who were subsequently confirmed as having a temporary conductive impairment and assessed the contribution of the tympanometry tests in identifying middle ear disease at the initial diagnostic clinic appointment. The tympanometric graphs were categorised as being flat, showing a clear peak with a compliance of $0.2 \mathrm{ml}$ or greater, or being indeterminate or unsuccessfully completed. A double peaked tympanogram was considered to be normal at this age.

\section{Parental recognition of deafness}

We also assessed parents' ability to recognise the presence of a hearing impairment after failure of the ABR test. At the initial diagnostic clinic follow up appointment after the ABR each parent was asked to respond to a written questionnaire that included the question: "Do you have any concerns about your child's hearing?"

\section{Results}

THE NUMBER OF ABR TESTS

Of the 25199 neonates who received the TEOAE test, 916 (3.6\%) were referred for ABR. Figure 2 details the reasons for referral and the results by aggregated threshold category. Five hundred and ninety six $(2.4 \%)$ failed the TEOAE test bilaterally, and $1.5 \%$ failed on two occasions. Of the bilateral failures, $531(89.1 \%)$ received an ABR, with the remainder failing to attend. Two hundred and seventy one of the neonates $(1.1 \%)$ failed the TEOAE test unilaterally and an ABR was requested. Of the unilateral TEOAE failures, $236(87.1 \%)$ attended their ABR appointment. Forty nine $(0.19 \%)$ of the cohort bypassed the 
Table 2. The value of the auditory brainstem response in predicting the presence of a permanent congenital hearing impairment

\begin{tabular}{lll}
\hline & \multicolumn{2}{l}{ Positive predictive value } \\
\cline { 2 - 3 } ABR threshold & $\begin{array}{l}\text { Bilateral screen } \\
\text { failure }\end{array}$ & $\begin{array}{l}\text { Unilateral screen } \\
\text { failure }\end{array}$ \\
\hline$\leqslant 20 \mathrm{dBnHL}$ & 0.000 & 0.000 \\
$30 \mathrm{dBnHL}$ & 0.007 & 0.000 \\
$40 \mathrm{dBnHL}$ & 0.01 & 0.000 \\
$50 \mathrm{dBnHL}$ & 0.08 & 0.000 \\
$60 \mathrm{dBnHL}$ & 0.32 & 0.028 \\
$70 \mathrm{dBnHL}$ & 0.60 & 0.25 \\
$\geqslant 80 \mathrm{dBnHL}$ & 1.00 & 1.00 \\
\hline
\end{tabular}

Values for bilateral screen failures are for the best hearing ear and for unilateral screen failures the worst hearing ear.

TEOAE screen, having been referred for diagnostic ABR by a paediatrician, or because of other parental or professional concerns. Of the 47 tested, 37 were at risk of deafness.

THE PREDICTIVE VALUE OF THE ABR

Thirty four of the 596 referred for TEOAE failure had a targeted bilateral PCHI. This represented $1.35 / 1000$ of the cohort tested and $6.4 \%$ of those receiving an $\mathrm{ABR}$ for bilateral TEOAE failure. Eleven had ABR thresholds $\geqslant 80 \mathrm{dBnHL}$ in their better ear (fig 2), with two having thresholds in the better hearing ear of $\leqslant 40 \mathrm{dBnHL}$. All of those with an ABR

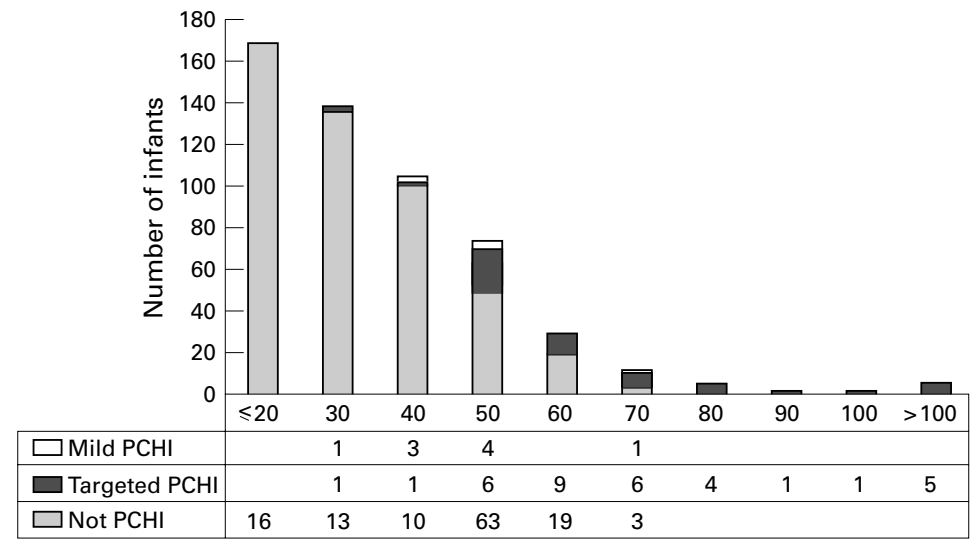

$A B R$ threshold in $\mathrm{dBnHL}$ in the better hearing ear

Figure 3 The presence of a permanent congenital hearing impairment (PCHI) and the auditory brainstem response $(A B R)$ thresholds achieved in early infancy for those failing the transient evoked oto-acoustic emission (TEOAE) screening test in both ears $(n=531)$.

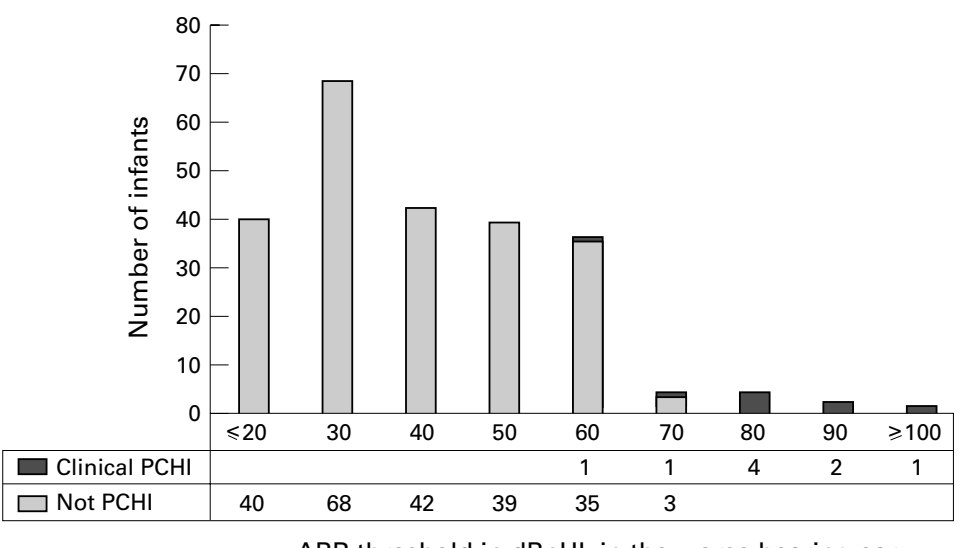

$A B R$ threshold in $\mathrm{dBnHL}$ in the worse hearing ear

Figure 4 The presence of a clinical permanent congenital hearing impairment (PCHI) and the auditory brainstem response (ABR) thresholds achieved for those failing the transient evoked oto-acoustic emission (TEOAE) screening test unilaterally $(n=236)$. threshold $\geqslant 80 \mathrm{dBnHL}$ had a targeted PCHI (PPV of 1). The PPV reduced with a lowering of the ABR threshold (table 2). In addition, nine of those referred for ABR were subsequently confirmed with a mild PCHI (yield of 0.36/1000). Figure 3 shows their ABR thresholds in the better hearing ear. Five of them had an $\mathrm{ABR}$ threshold in the better hearing ear of $>40 \mathrm{dBnHL}$.

Nine of the 271 who failed the TEOAE test unilaterally had clinical evidence of a permanent unilateral hearing impairment (fig 4). This was $0.36 / 1000$ of the overall cohort, and $3.8 \%$ of those receiving an ABR for unilateral TEOAE failure. Two with hearing thresholds $<80 \mathrm{dBnHL}$ had permanent conductive impairments, with one having a clinically apparent external ear malformation. As in the bilateral impairments, we considered all those with an ABR threshold $\geqslant 80 \mathrm{dBnHL}$ on clinical and audiological grounds to have a unilateral permanent deafness (PPV of 1) (table 2).

From the small number of direct ABR referrals there was a yield of three with a targeted PCHI. This represented $0.12 / 1000$ of the overall tested cohort, and $6.4 \%$ of those receiving an ABR from this referral route. Therefore, the "pick up" rate was the same as that for ABRs undertaken for bilateral TEOAE failure. Two were referred because they had branchial arch syndromes with external auditory canal atresias. The other had an auditory neuropathy with neonatal TEOAEs being present.

LONGITUDINAL ANALYSIS OF ABR TESTS

Figure 2 shows the overall numbers receiving an ABR test from 1992 to 1997. Figure 5 details the results of the longitudinal analysis by year. Over this five year period, 22487 infants received the TEOAE screen. Of these, $501(2.23 \%)$ failed the screen bilaterally and were referred for an ABR. Of these, 115 infants (4.6/1000 screened) were confirmed, with an ABR threshold $>40 \mathrm{dBnHL}$. There were no significant differences in the annual yield of these infants $\left(\chi^{2}, 0.217\right.$; degrees of freedom (df), $4 ; \mathrm{p}>0.50$ ). Most (105 of 115) were confirmed with an ABR threshold $>40 \mathrm{dBnHL}$ and $<80 \mathrm{dBnHL}$ in the better hearing ear. During this period, an additional 224 infants were referred for an $A B R$ because of a unilateral TEOAE failure $(1.0 \%$ of those screened). Of these, 82 (3.25/1000 screened) had a unilateral ABR impairment $>40 \mathrm{dBnHL}$ in the worst hearing ear. Once again this yield did not alter significantly from year to year $\left(\chi^{2}\right.$, $0.965 ; \mathrm{df}, 4 ; \mathrm{p}>0.50)$. However, fig 5 confirms that there was a dramatic fall in those who failed the TEOAE test bilaterally and who then passed the ABR with thresholds of $\leqslant 40 \mathrm{dBnHL}$ in the better hearing ear. Overall during the five years, 386 infants fell into this category (17/1000 screened). The yield was $23 / 1000(n=307)$ in the first three years and $8.6 / 1000(n=79)$ in the latter two years. This large reduction in those who had failed the TEOAE tests but had normal hearing in their better hearing ear (or at worst a mild hearing impairment), was highly significant $\left(\chi^{2}\right.$, 64.435; df, $1 ; \mathrm{p}<0.001)$. 


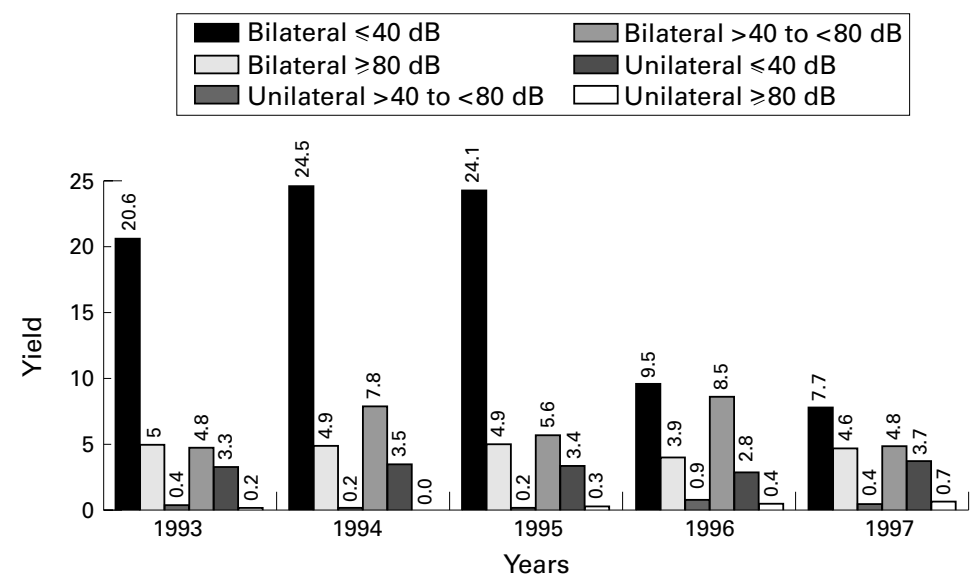

Figure 5 The annual number of infants requiring an auditory brainstem response (ABR) test and the results from 1993 to 1997 (yields/1000 screened).

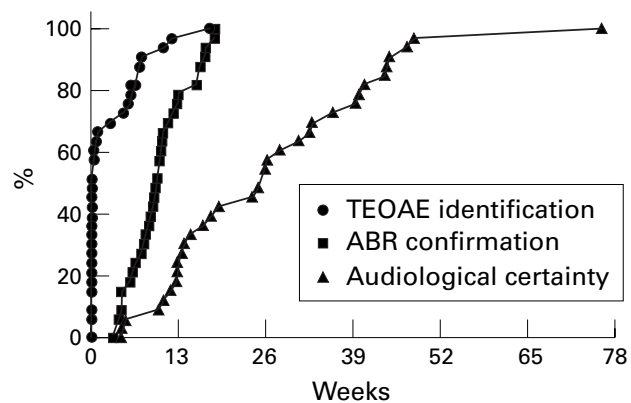

Figure 6 The cumulative distributions of outcome ages for those identified by the universal neonatal screen $(n=34)$. $A B R$, auditory brainstem response; TEOAE, transient evoked oto-auditory emission.

Table 3 Outcome ages and delays for the bilateral screen failures

\begin{tabular}{|c|c|c|c|c|c|}
\hline & \multicolumn{3}{|c|}{$A B R>40$ to $<80 \mathrm{dBnHL}$} & \multicolumn{2}{|c|}{$A B R \geqslant 80 d B n H L$} \\
\hline & $\begin{array}{l}\text { Mild } \\
\text { PCHI }\end{array}$ & $\begin{array}{l}\text { Moderate } \\
\text { PCHI }\end{array}$ & No PCHI & $\begin{array}{l}\text { Moderate } \\
\text { PCHI }\end{array}$ & $\begin{array}{l}\text { Severel } \\
\text { profound } \\
\text { PCHI }\end{array}$ \\
\hline \multicolumn{6}{|c|}{ Age of TEOAE (weeks) } \\
\hline Number & 5 & 21 & 85 & 2 & 9 \\
\hline Mean & 1.57 & 1.53 & 3.29 & 6.1 & 2.76 \\
\hline SD & 3.22 & 2.95 & 4.52 & 1.7 & 4.26 \\
\hline 25 th centile & 0.13 & 0.09 & 0.1 & $\mathrm{~N} / \mathrm{A}$ & 0.08 \\
\hline 50th centile & 0.14 & 0.23 & 0.3 & 6.1 & 0.19 \\
\hline \multicolumn{6}{|c|}{ Age of $A B R$ (weeks) } \\
\hline Number & 5 & 21 & 85 & 2 & 9 \\
\hline Mean & 9.46 & 10.13 & 11.30 & 14.28 & 8.29 \\
\hline SD & 3.18 & 4.62 & 4.07 & 3.84 & 2.72 \\
\hline 25 th centile & 7.71 & 6.43 & 9.14 & N/A & 6.15 \\
\hline 50th centile & 9.14 & 10.00 & 10.71 & 14.28 & 8.3 \\
\hline \multicolumn{5}{|c|}{ Age of certainty (weeks) } & 9.93 \\
\hline Number & 5 & 21 & 74 & 2 & 9 \\
\hline Mean & 46.34 & 31.51 & 35.83 & 18.5 & 11.92 \\
\hline SD & 33.14 & 15.89 & 8.87 & 0.71 & 4.24 \\
\hline 25 th centile & 24.28 & 25.00 & 30.96 & N/A & 8.50 \\
\hline 50th centile & 32.57 & 31.00 & 35.43 & 18.5 & 13.01 \\
\hline 75th centile & 48.86 & 40.00 & 39.71 & N/A & 14.50 \\
\hline \multicolumn{6}{|c|}{ Delay TEOAE to $A B R$ (weeks) } \\
\hline Number & 5 & 21 & 85 & 2 & 9 \\
\hline Mean & 7.88 & 8.6 & 8.01 & 8.18 & 5.53 \\
\hline SD & 3.39 & 3.88 & 4.76 & 2.14 & 2.70 \\
\hline 25th centile & 5.1 & 5.93 & 4.27 & N/A & 3.47 \\
\hline 50th centile & 7.57 & 8.55 & 7.29 & 8.18 & 4.77 \\
\hline 75th centile & 9.08 & 10.5 & 10.95 & N/A & 7.99 \\
\hline \multicolumn{6}{|c|}{ Delay ABR to certainty (weeks) } \\
\hline Number & 5 & 21 & 74 & 2 & 9 \\
\hline Mean & 38.35 & 21.38 & 24.54 & 4.21 & 3.63 \\
\hline SD & 33.66 & 15.65 & 8.75 & 3.13 & 2.67 \\
\hline 25 th centile & 19.43 & 12.43 & 18.07 & N/A & 0.43 \\
\hline 50th centile & 19.62 & 21.57 & 25.86 & 4.21 & 4.4 \\
\hline 75th centile & 43.57 & 30.86 & 27.98 & N/A & 5.72 \\
\hline
\end{tabular}

DELAYS IN ACHIEVING AUDIOLOGICAL CERTAINTY Figure 6 shows the distributions of the outcome ages for the 34 infants with a targeted PCHI identified by the universal neonatal screen. It confirms that two thirds of those with a PCHI were identified within the 1 st week of life. Three quarters of ABR confirmations were by the 3rd month of life, with all being undertaken by 18 weeks. Although the figure shows a lag after the ABR, audiological certainty had still been achieved for most infants within the first half of infancy (median, 25 weeks), with two thirds having achieved this outcome by 8 months of age, and all but one by their $1 \mathrm{st}$ birthday.

Table 3 details the average outcome ages for those failing the TEOAE screen bilaterally and with an ABR threshold $>40 \mathrm{dBnHL}$ in the better hearing ear. The ages were analysed by the different ABR categories and by the audiological diagnosis when achieved with certainty. Of the 122 who failed the TEOAE test bilaterally and had ABR thresholds $>40 \mathrm{dBnHL}, 85$ were considered to have a temporary conductive impairment, without an underlying PCHI. They all had ABR thresholds $>40 \mathrm{dBnHL}$ and $<80 \mathrm{dBnHL}$. The absence of a PCHI was only confirmed with certainty in 74 of them. Of the 11 others, seven had moved away, and four were persistent non-attenders. Inspection of their records and contact with the primary care teams failed to reveal a PCHI in any.

Thirty seven of the infants had a PCHI. Eleven of those with a PCHI had an ABR threshold $\geqslant 80 \mathrm{dBnHL}$. Nine of them had a severe or profound PCHI confirmed with certainty by the diagnostic assessments. Two had confirmed moderate sensori-neural hearing impairments, with the degree of deafness being somewhat less severe than had been suggested by the initial ABR. They had mixed hearing impairments with superimposed middle ear effusions in infancy. None of those with $\geqslant 80 \mathrm{dBnHL}$ ABR thresholds had either a mild PCHI or a hearing loss entirely attributable to middle ear effusions. Of those with ABR thresholds $>40 \mathrm{dBnHL}$ and $<80 \mathrm{dBnHL}, 26$ had a PCHI. All had a mild or moderate PCHI when the deafness was confirmed with audiological certainty. Once again, the initial ABR had overestimated the degree of deafness in five of these infants, who actually had only a mild PCHI. They also had superimposed middle ear effusions established in the first half of infancy.

The infants who had failed the ABR with a threshold $>40 \mathrm{dBnHL}$ in the better hearing ear could therefore be aggregated into the five different groups detailed in table 3. The median delays from the initial TEOAE test to the ABR ranged from five to eight weeks for all groups. However, the delays from the ABR test to the age of audiological certainty was much more variable. The median age of audiological certainty for the three subgroups with ABR thresholds $>40 \mathrm{dBnHL}$ and $<80 \mathrm{dBnHL}$ varied between 31 and 35 weeks. Those with this ABR threshold who had a PCHI had their deafness confirmed with certainty slightly before those who were found to have no permanent loss. The median delays from ABR 


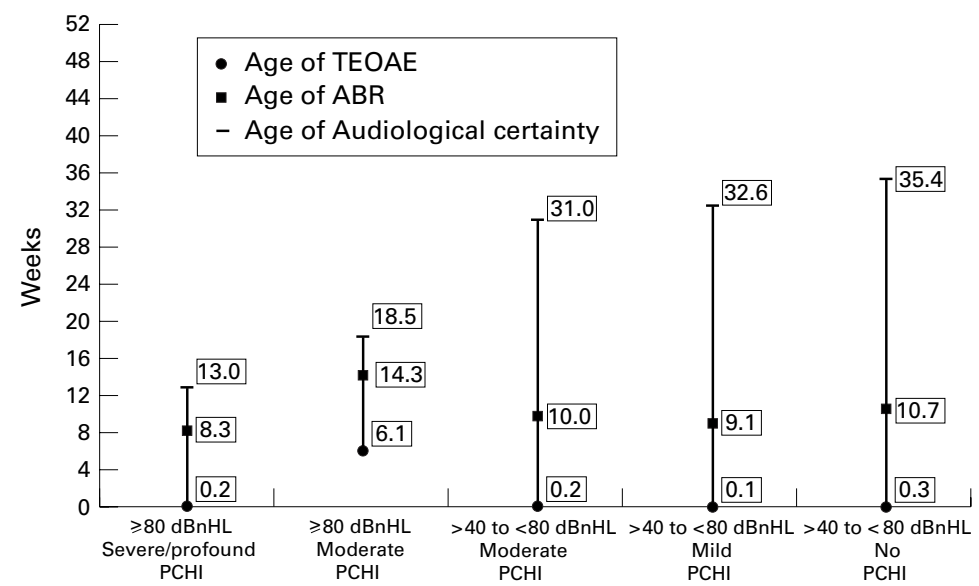

Figure 7 The median ages in weeks of transient evoked oto-auditory emission (TEOAE) identification, the auditory brainstem response (ABR) test, and achievement of audiological certainty for those failing the ABR bilaterally. PCHI, permanent congenital hearing impairment.

to certainty for these groups with an $\mathrm{ABR}$ threshold $>40$ and $<80 \mathrm{dBnHL}$ varied from 20 to 26 weeks. They were compared by transforming the time delays to ranks and applying a Mann Whitney U test. There were no significant differences in the delays experienced by the three groups with ABR thresholds $>40 \mathrm{dBnHL}$ and $<80 \mathrm{dBnHL}$, irrespective of the type of the loss eventually found to be present ( $p>0.10$ for all comparisons).

Nine of the 11 with a bilateral ABR hearing threshold of $\geqslant 80 \mathrm{dBnHL}$ eventually turned out to have either a severe or profound PCHI, with two having a mixed deafness and a moderate degree of PCHI. Those who were eventually diagnosed as having a severe or profound deafness achieved full diagnosis at an average age of 12 weeks. The two who had an ABR threshold of $\geqslant 80 \mathrm{dBnHL}$, but who eventually were found to have a moderate PCHI, both had additional disabilities, and treatment for additional illnesses delayed their initial identification and ABR confirmation. The mixed nature of their hearing losses was diagnosed with certainty at an average age of 5 months. For all those with an initial ABR result of $\geqslant 80 \mathrm{dBnHL}$, the median delay from $\mathrm{ABR}$ to certainty was just four weeks. When compared with the lag achieved for those with an ABR threshold > $40 \mathrm{dBnHL}$ and $<80 \mathrm{dBnHL}$, this reduction was highly significant (Mann Whitney test, mean ranks 61 and $10 ; \mathrm{p}<0.001)$. Figure 7 summarises the median outcome ages and delays.

For reasons detailed above, the delay in achieving audiological certainty was even greater for the children referred for an $A B R$

Table 4 The responses to the question: "Do you have any concerns about your child's hearing?"

\begin{tabular}{lcrrr}
\hline & Number & Yes & No & Uncertain \\
\hline ABR better hearing ear $>40$ to $<80$ dBnHL & & & & \\
$\quad$ Mild PCHI & 5 & 1 & 4 & 0 \\
Moderate PCHI & 20 & 4 & 14 & 2 \\
No PCHI & 74 & 2 & 68 & 4 \\
Subtotal & 99 & 7 & 86 & 6 \\
ABR better hearing ear $\geqslant 80$ dBnHL & 11 & 7 & 1 & 3 \\
Total & 110 & 14 & 87 & 9 \\
\hline
\end{tabular}

because of unilateral TEOAE failure. Although the ABR thresholds, and subsequent clinical examinations, were all consistent with nine having a unilateral PCHI, only three had reached an age when a fully masked pure tone audiogram had been achieved. The median age of audiological certainty of the three infants was 188 weeks, representing a lag of 181 weeks from the ABR.

Similarly, parents of the 409 infants who had a hearing threshold of $\leqslant 40 \mathrm{dBnHL}$ in the better hearing ear at the ABR were reassured that early habilitation was not required. However, the children were given an appointment for a behavioural test of hearing later in infancy; after this, two children were confirmed to have a targeted PCHI, and four had mild PCHI. There has been subsequent evidence that the hearing impairments of those who "passed" the ABR, but had a moderate PCHI, have been mildly progressive, but in both cases the shape of the audiogram in the better hearing ear also contributed to the fact that the hearing impairment across the frequency range was not truly reflected by the ABR in early infancy. Fortunately, the fact that they were both considered to have passed the ABR did not delay their age of diagnosis compared with the others with a similar degree of hearing impairment who were identified from the ABR. For both there was certainty about the presence of a targeted deafness by the age of 44 weeks, with a mean delay of 29 weeks from the ABR test.

TYMPANOMETRY

We examined tympanometry results for the 85 infants without a PCHI who had nevertheless failed the TEOAE test bilaterally and been referred to diagnostic clinics with an $A B R$ threshold $>40 \mathrm{dBnHL}$. Of the 170 ears tested, indeterminate results were obtained from 83 $(49 \%)$. Of the 87 where a valid result was obtained, 35 had tympanometric traces showing peaks of $0.2 \mathrm{ml}$ or greater. In an older child, all these traces would have been consistent with the presence of normally aerated middle ear clefts-but in fact, all 35 of these ears had TEOAEs that failed to meet the pass/fail criteria and $\mathrm{ABR}$ thresholds $>40 \mathrm{dBnHL}$. The remaining 52 ears had flat traces.

THE PRESENCE OF PARENTAL CONCERNS

Table 4 details the responses to the question: "Do you have any concerns about your child's hearing?" Parents of 99 of 111 infants with ABR thresholds $>40 \mathrm{dBnHL}$ and $<80 \mathrm{dBnHL}$ in the better hearing ear responded. Only seven replied that they did have concerns, with six being uncertain, and the remaining 86 having no concerns. Of the 11 with a severe or profound ABR impairment, seven parents confirmed concerns at the diagnostic assessment appointment after the ABR test. Of the remainder, three were uncertain.

\section{Discussion}

The possibility of introducing universal neonatal hearing screens has focused attention on the ability to confirm the diagnosis of deafness in those failing the screening tests. Such attention 
is overdue. The worth of early identification is dictated by the ability to implement early habilitation. Without the latter, the former can hardly be justified. Yet, problems associated with diagnostic audiological testing during the early months of infancy have been widely experienced since selective neonatal screens were introduced in the UK in the 1980s. A survey undertaken as part of the critical review of the role of neonatal hearing screening in the detection of congenital hearing impairment confirmed that about three quarters of districts in the UK had some sort of selective neonatal screen in place. ${ }^{1}$ They have relied upon a basic air conduction ABR to click stimuli as the basis of the confirmatory examination. However, restricting this examination to measuring sensitivity to air conduction stimuli has obvious limitations and is completely unacceptable for older children. Diagnosing the type of hearing impairment requires additional test methods, but such tests have not been used routinely at this age. Confirmation of permanent deafness with "audiological certainty" might be achieved only by undertaking further tests as the infant matures.

We have shown the resulting delays in those infants with a hearing impairment identified by the Whipps Cross universal neonatal screen. From the screened cohort of just over 25000 neonates, there was a targeted yield of $1.35 / 1000$. This represented $1.18 / 1000$ of the cohort eligible for the screen. An additional nine $(0.36 / 1000$ of the cohort screened) had a mild PCHI, with the same number having a unilateral PCHI.

Those receiving an ABR test had failed their initial TEOAE test at a median age of under 1 week. The median age of the ABR was delayed to between 2 and 3 months with the median lag varying between 5 and 9 weeks. The delay between the initial TEOAE failure and the $A B R$ varied slightly, depending upon the $A B R$ subgroup examined. Such delays could be predicted from the programme protocol, which required the TEOAE test to be repeated before proceeding to the more difficult and time consuming ABR test.

Three quarters of the infants receiving an ABR for bilateral TEOAE failure passed the ABR test with a $40 \mathrm{dBnHL}$ or better threshold in the better hearing ear. However, any such threshold pass/fail criterion is arbitrary and subsequent follow up of the cohort confirmed that at least four of these infants did indeed have a mild permanent deafness, with a further two having a moderate impairment. Complete ascertainment of those with a mild degree of deafness will not be achieved until the entire cohort has been tested by pure tone audiometry in school. It is also possible that a very small number who passed the ABR using this criterion might have a moderate PCHI, which has not been confirmed to date. However, although we showed that a small number of those falling within this category might have a permanent hearing impairment, most do not require intervention at this age. The parents received this reassurance on average less than two months after the first TEOAE test failure.
Importantly, there were far fewer of these during the period studied. In 1996, the percentage of infants who required an ABR for bilateral screen failure fell from $2.9 \%$ to $1.4 \%$. Those who were referred for ABR but had thresholds of $40 \mathrm{dBnHL}$ or better fell from $2.4 \%$ to $<1 \%$. This reduction was maintained during the next year (and subsequently). It is an important improvement because this small percentage represents the screen false positives. It might have been attributable to several programme changes, with the main one being a relaxation of the stringent pass/fail criterion after the first three year screen evaluation.

However, a major concern was the delay between failing the screen and having deafness confirmed. In all those with a hearing impairment identified by the ABR there were delays before the type of hearing impairment was confirmed with certainty. Eleven of the cohort $(0.4 / 1000)$ were identified with an ABR threshold $\geqslant 80 \mathrm{dBnHL}$. Follow up confirmed that they all had a permanent hearing impairment, with nine of them having severe or profound deafness. This was recognised after a median delay of just over one month. Even this delay was not a period of inactivity. It allowed the teacher time to undertake a home visit, and the baby to be given an appointment at the diagnostic clinic. Once again this could be predicted by the programme protocol. The problems lay in those infants with an $\mathrm{ABR}$ threshold $>40 \mathrm{dBnHL}$ and $<80 \mathrm{dBnHL}$ in the better hearing ear. Just under $5 / 1000$ of the tested cohort failed the TEOAE test bilaterally and had such an impairment. This remained constant throughout the period of the study, and was unaffected by programme changes introduced during the final two years. The PPV of the ABR varied according to the threshold achieved in the better hearing ear. Thus, $60 \%$ of those with a threshold of $70 \mathrm{dBnHL}$, but only $8 \%$ of those with a $50 \mathrm{dBnHL}$ threshold, had a PCHI. Of 111 infants with ABR thresholds between $>40 \mathrm{dBnHL}$ and $<80 \mathrm{dBnHL}$, 26 had a PCHI; of these, five were eventually confirmed with a mild impairment not targeted for detection by the screen. Therefore, the infants in this group all required a reliable assessment of the type and degree of loss before they could be referred confidently to the multidisciplinary habilitation clinics. These babies presented the greatest audiological uncertainty. Although the median age of the ABR was 9 or 10 weeks, there was a lag of several months before the type of hearing loss was diagnosed. Most (85 of 111) had middle ear effusions without a permanent component to their impairment. Their effusions either resolved or were managed by surgical middle ear ventilation. However, on average it took almost 26 weeks to confirm that there was no underlying PCHI. Those who did have a PCHI fared little better. For those with a mild PCHI it took 20 weeks from the ABR before the permanence of the impairment was confirmed, with those with a moderate impairment experiencing an average delay of 22 weeks.

Even longer delays were experienced in the nine infants identified with a unilateral PCHI. 
After a masked ABR, traditional behavioural tests of hearing cannot sensitively separate the ears. For this, visual reinforcement audiometry with ear inserts is required. At the outset of the programme such a visual reinforcement audiometry test rig was not available to those identified with a unilateral impairment-and the median delay from the ABR to confirmation by masked audiogram was over 180 weeks.

The results confirmed that the traditionally used air conduction ABR was a satisfactory tool for specifying the management of those infants with a severe/profound hearing impairment, or normal/near normal hearing. Fortunately, this comprises most of the infants receiving the $\mathrm{ABR}$, and it allowed the follow up management protocol illustrated in fig 1 to be used effectively. The protocol was further refined with the results from our study. The predictive value of an ABR threshold $\geqslant 80 \mathrm{dBnHL}$ is very high, and immediate referral for habilitation is possible. Similarly, the chance of an infant with an ABR threshold $\leqslant 40 \mathrm{dBnHL}$ having a targeted PCHI is very low. Although some sort of ongoing hearing surveillance is required to catch the small numbers with a progressive sensori-neural deafness, or an unusually configured audiometric shape, parents can be confidently reassured. Difficulties arose with the group detailed in the original management strategy (fig 1) as having failed the $\mathrm{ABR}$ with a $>40 \mathrm{dBnHL}$ threshold but in whom the audiological assessment diagnosed a "less than severe hearing impairment".

A parent centred approach to establishing the need for habilitation was used, with the opportunity for home visiting by the teacher of the deaf. However, parents were not able to identify the need for habilitation. Although the parents were all aware of the ABR result, and were observing reactions to sounds within the home, only $7 \%$ of those whose baby had ABR thresholds that were moderately impaired had any concerns about their hearing, with a further $6 \%$ being uncertain. The remainder had no concerns. Although the central role of the family in effectively managing their hearing impaired child cannot be overemphasised, these results caution against empowering parents to establish the diagnosis at this age by behavioural observations. Partnership with parents is vital, but this can only be diagnostically useful if much more sensitive behavioural indicators are available. In practice, there needs to be unequivocal professional confirmation that habilitation is required.

At the outset of the programme confirmation after the ABR was by behavioural observation audiometry and tympanometry. The absence of both reflexive and attentive behaviours helped to show the presence of a severe or profound deafness. However, in those with a moderate impairment, the retention of reflexive behaviours combined with the inherent difficulties in observing the absence of attentive behaviours to quieter sounds prevented the effective use of behavioural observation audiometry.

\section{Key messages}

- Useful implementation of universal neonatal hearing screens depends on the ability to confirm both the type and degree of deafness in early infancy

- Screening may be relatively straightforward but audiological diagnosis at this age is not

- Severe or profound deafness can be identified in early infancy with minimal delay but moderate deafness is confirmed on average at 8 months

- Targeting moderate deafness in early infancy demands that audiological tests are developed to confirm a permanent hearing impairment in those identified by the screen

Tympanometry using a $226 \mathrm{~Hz}$ probe tone was not useful for identifying whether young infants with moderately impaired ABR thresholds had middle ear effusions. Almost half of the tympanometric traces were indeterminate. This result mirrors that achieved elsewhere, with an earlier study having reported that one third of recordings from 600 ears of young babies were unsuccessful. ${ }^{16}$ Nevertheless, conventional tympanometry is still used for testing neonates. ${ }^{8}$ We encountered an additional problem. The tympanometric test was applied to a group of young infants who had failed the neonatal screen. Follow up confirmed that most of this group had either an external or middle ear disorder at the time of the examination, yet $40 \%$ of them had normal tympanometric results. We believe there is a good case for abandoning the use of low probe tone tympanometry. However, although there have been useful and informative studies of the use of high frequency probe tone tympanometry in neonates, a satisfactory classification of the results is still not available. This was introduced in the latter part of our study, and will be the subject of another report. During our study there was no satisfactory way of determining the presence of middle ear effusion by tympanometric examination undertaken in the diagnostic assessment after ABR failure.

The analysis confirmed that a test battery is required at this age, which allows the diagnosis of both the type and degree of deafness. It includes bone conduction testing, high frequency probe tone oto-admittance measurements, and a clinical examination of middle ear status. Characterisation of the shape of the hearing impairment would also offer advantages when fitting hearing aids.

Despite the absence of such a battery at the outset, definite benefits resulted from the programme. Those with severe or profound impairment had their deafness confirmed with certainty at an average age of approximately 3 months, and more than half of those with a moderate deafness had their impairment confirmed by 8 months - the age at which they would hitherto have received the distraction 
test. Early habilitation is therefore still possible in programmes undertaking both universal and selective neonatal screening, even in the absence of highly developed test batteries. In addition, earlier identification will almost certainly be the catalyst for improvements in early diagnostic procedures, and the current debate has already prompted a resurgence of such endeavour in the UK. A national working party has been convened to explore the current difficulties. Clearly, it is vital that methods to achieve this audiological certainty continue to be developed. It would be difficult to justify the aim of neonatal identification of those with anything less than a severe or profound bilateral deafness if the impairments were only confirmed towards the end of the 1 st year of life.

1 Davis A, Bamford J, Wilson I, Ramkalawan T, Forshaw M, Wright S. A critical review of the role of neonatal hearing screening in the detection of congenital hearing impairscreening in the detection of congenital hearing
ment. Health Technol Assess 1997;1(10):1-196.

2 Tucker SM, Bhattacharya J. Screening hearing impairment in the newborn using the auditory response cradle. Arch Dis in the newborn using the

3 Watkin PM. Neonatal otoacoustic emission screening and the identification of deafness. Arch Dis Child Fetal Neonatal Ed 1996;74:F16-25.
4 Watkin PM. Outcomes of neonatal screening for hearing loss by otoacoustic emission. Arch Dis Child Fetal Neonatal Ed 1996;75:F158-68.

5 Watkin PM, Nanor J. The implications for educational services of universal neonatal hearing screening. Deafness and Education 1997;21:19-33.

6 McLellan MS, Webb CH. Ear studies in the newborn infant. F Paediatr 1961;58:523-7.

7 Shurin PA, Pelton SI, Kinkelstein J. Tympanometry in the diagnosis of middle ear effusion. $N$ Engl f Med 1977;24: $412-17$

8 El-Refaie A, Parker DJ, Bamford J. Otoacoustic emission versus ABR screening: the effect of external and middle ear abnormalities in a group of SCBU neonates. $\mathrm{Br} \mathcal{F}$ Audiol 1996;30:3-8.

9 Weatherby LA, Bennett HD. The neonatal acoustic reflex. Scand Audiol 1980;9:103-10.

10 Sutton GJ, Gleadle P, Rowe S. Tympanometry and otoacoustic emissions in a cohort of special care neonates. Br F Audiol 1996;30:9-17.

11 Stapells DR, Oates P. Estimation of the pure tone audiogram by the auditory brainstem response: a review. Audiol Neurootol 1997;2:257-80.

12 Thompson M, Thompson G. Responses of infants and young children as a function of auditory stimuli and test methods. Speech and Hearing Research 1972;15:699.

13 Marchant CD, Shurin PA. Otitis media in the first year of life. Ann Otol Rhinol Laryngol 1990;99(suppl 149):33-4.

14 Ewing IR, Ewing AWG. The ascertainment of deafness in infancy and early childhood. F Laryngol Otol 1944;59: 309-38.

15 McCormick B. Hearing screening by health visitors; a critical appraisal of the distraction test. Health Visit 1983;56: 449-51.

16 Thornton ARD, Kimm L, Kennedy CR, Cafarelli Dees D. External and middle ear factors effecting the interpretation of transient evoked otoacoustic emissions in neonatal hearing screening. Br f Audiol 1993;27:319-27. 